\title{
Utility of nonpenetrating titanium clips for dural closure during spinal surgery to prevent postoperative cerebrospinal fluid leakage
}

\author{
Kiyoshi Ito, MD, Tatsuro Aoyama, MD, Tetsuyoshi Horiuchi, MD, and Kazuhiro Hongo, MD \\ Department of Neurosurgery, Shinshu University School of Medicine, Matsumoto, Japan
}

OBJECT The nonpenetrating titanium clip has been successfully used in peripheral arterial bypass surgery. The purpose of this study was to evaluate the leakage pressures and patterns of nonpenetrating titanium clips using a simple model that mimicked spinal surgery. In addition, the authors describe their surgical experience with these clips and the follow-up results in 31 consecutive patients.

METHODS The authors compared nonpenetrating titanium clips and expanded polytetrafluoroethylene (ePTFE) sutures in relation to the water pressure that could be tolerated by sutured ePTFE sheets, and the leakage pressure patterns were determined. The changes in leakage pressures at 5 minutes, 30 minutes, and 12 hours were examined when the clips and sutures were used in combination with the mesh-and-glue technique in an in vitro study. Thirty-one patients underwent spinal intradural procedures using nonpenetrating titanium clips to suture the dura maters using the meshand-glue technique, involving fibrin glue and polyglycolic acid-fibrin sheets.

RESULTS A significant difference was apparent between the ePTFE suture group and the nonpenetrating titanium clip group, with the latter showing a leakage pressure that could be sustained and was $1508 \%$ higher than that of the former $(p=0.001)$. In relation to leakage patterns, the nonpenetrating titanium clips did not make any suture holes in the ePTFE sheet and fluid leakage occurred between the clips, whereas fluid leakage was associated with the pressure elevation that occurred at the suture holes made by the ePTFE sutures. Of the 31 patients who underwent spinal intradural procedures using nonpenetrating titanium clips, 1 (3.2\%) experienced cerebrospinal fluid (CSF) leakage postoperatively. № other complications-for example, allergic reactions, adhesions, or infections-were encountered.

CONCLUSIONS The interrupted placement of nonpenetrating titanium clips enables dural closure without creating any holes. These clips facilitate improvements in the initial leakage pressure and reduce postoperative CSF leakage following spinal surgery. The authors conclude that it is very beneficial to suture the spinal dura mater using nonpenetrating titanium clips given the anatomical characteristics of the spinal dura mater and the fact that the clips do not create suture holes.

http://thejns.org/doi/abs/10.3171/2015.3.SPINE141215

KEY WORDS cerebrospinal fluid leakage; dural closure; intradural lesion; nonpenetrating clips; spinal surgery; technique

$\mathrm{P}$ OSTOPERATIVE cerebrospinal fluid (CSF) leakage is a major surgical complication in spinal surgery. It is critical to prevent CSF leakage, especially after surgery for spinal intradural lesions. Several sealing materials have been proposed as adjuncts to sutured dural closures to reduce the potential for adverse events, such as infection, and to lower the risk of pseudomeningocele formation. However, CSF leakage is not always prevented during these procedures. Compared with the intracranial region, the spinal region is a narrower operative field, and the dura mater is thinner and more fragile $;{ }^{17}$ hence, meticulous dural suturing can be impossible after intradural procedures.

The nonpenetrating titanium clip is designed to create faultless vascular anastomoses. This clip does not create any suture holes, and it has been successfully used in peripheral arterial bypass surgery. $3,5,6,8,15,20$ The device was approved by the US Food and Drug Administration for

ABBREVIATIONS CSF = cerebrospinal fluid; ePTFE = expanded polytetrafluoroethylene; PGA = polyglycolic acid; VCS = vessel closure system .

SUBMITTED December 1, 2014. ACCEPTED March 26, 2015.

INCLUDE WHEN CITING Published online August 28, 2015; DOI: 10.3171/2015.3.SPINE141215. 
vascular anastomosis and reconstruction in December 1993 and for dural closure in 1996.

Animal studies using nonpenetrating titanium clips for duraplasty have demonstrated significantly less inflammation, lower levels of reaction to foreign bodies, and fewer meningoneural adhesions than those with penetrating needles and sutures. ${ }^{11}$ However, no published experimental or clinical studies have described the usefulness of nonpenetrating titanium clips for the prevention of postoperative CSF leakage. We have applied this clip to closure of the dura mater following surgery to remove intradural spinal lesions. This is the first experimental study to investigate the utility of nonpenetrating titanium clips in the prevention of postoperative CSF leakage.

The purpose of this study was to evaluate the leakage pressures and patterns associated with nonpenetrating titanium clips by using a simple model that mimicked spinal surgery. In addition, we describe our surgical experience with these clips and the follow-up results in 31 consecutive patients.

\section{Methods}

\section{Nonpenetrating Titanium Clips}

Nonpenetrating titanium clips (vessel closure system [VCS] clip; LeMaitre Vascular Inc.) are produced for the reconstruction of tubular organs, including peripheral arteries, veins, and the biliary tract. The technical and biological advantages of nonpenetrating titanium clips have been described previously. ${ }^{3,5,6,8,20}$ Scanning electron microscopy shows that the clip tips do not completely close; consequently, no suture holes are created (Fig. 1). The clip applicator allows repetitive suturing using 20 preloaded clips (Fig. 2).

\section{Experimental Study: Initial Leakage Pressures During Pressure Leakage Tests}

We designed experiments to estimate the water pressures that could be tolerated by sutured expanded polytetrafluoroethylene (ePTFE) sheets (Flex USCI ePTFE sheet, USCI Japan Ltd.). Our experimental device consisted of a plastic cup filled with water, which contained a 30-mmdiameter hole, a digital manometer, and a pressure syringe connected by a tube to the water cup (Fig. 3). The size of the hole, which was $30 \mathrm{~mm}$, was considered to mimic the length of 1 vertebra. The top of the plastic cup was covered with ePTFE sheets that were sutured with 2 materials, namely, the nonpenetrating titanium clips that were placed at intervals of $2 \mathrm{~mm}$ or ePTFE running sutures (Gore-Tex suture CV-5, Japan Gore-Tex Inc.) that were placed at intervals of $2 \mathrm{~mm}$ (Fig. 4). To minimize leakage from the needle holes created during dural suturing, the ePTFE sutures were prepared with a needle to thread ratio of 1:1. The leakage pressures were measured for 2 different leakage patterns using the ePTFE sutures and the nonpenetrating titanium clips.

We measured the leakage pressures for each suture group using pressure leakage tests $(n=14$ tests for each group). The leakage patterns from the leakage points in each group were simultaneously observed in detail.

The mesh-and-glue technique has been described for the prevention of postoperative CSF leakage, ${ }^{9}$ and similar techniques have been reported by other authors. ${ }^{4,16}$ This method involves the use of an absorbable polyglycolic acid (PGA) mesh (Neoveil, Gunze Limited) soaked in fibrin glue (Beriplast P, CSL Behring), and in this study, the mesh was placed on and used to seal the suture lines in the ePTFE sheets (Fig. 5). This method is effective at reinforcing the suture lines. ${ }^{9}$ We investigated the efficacy of this method when it was used with intermittently placed VCS clips. The suture line consisting of the nonpenetrating titanium clips was covered with the absorbable PGA mesh soaked in fibrin glue, and we investigated the leakage pressures at 5 minutes, 30 minutes, and 12 hours.

\section{Clinical Study: Patient Population and Surgical Technique}

Thirty-one patients with spinal intradural lesions, including 24 with neurinomas, 3 with meningiomas, 2 with perimedullary arteriovenous fistulas, and 2 with tethered cord syndrome associated with spinal lipomas, were surgically treated, and the edges of the dura maters were approximated using nonpenetrating titanium clips at our hospital between July 2013 and August 2014. After the intradural procedures, the dura maters were sutured with the clips under the operative microscope using the mesh-andglue technique. All of the patients underwent MRI within 2 weeks of the operation to check for CSF leaks.
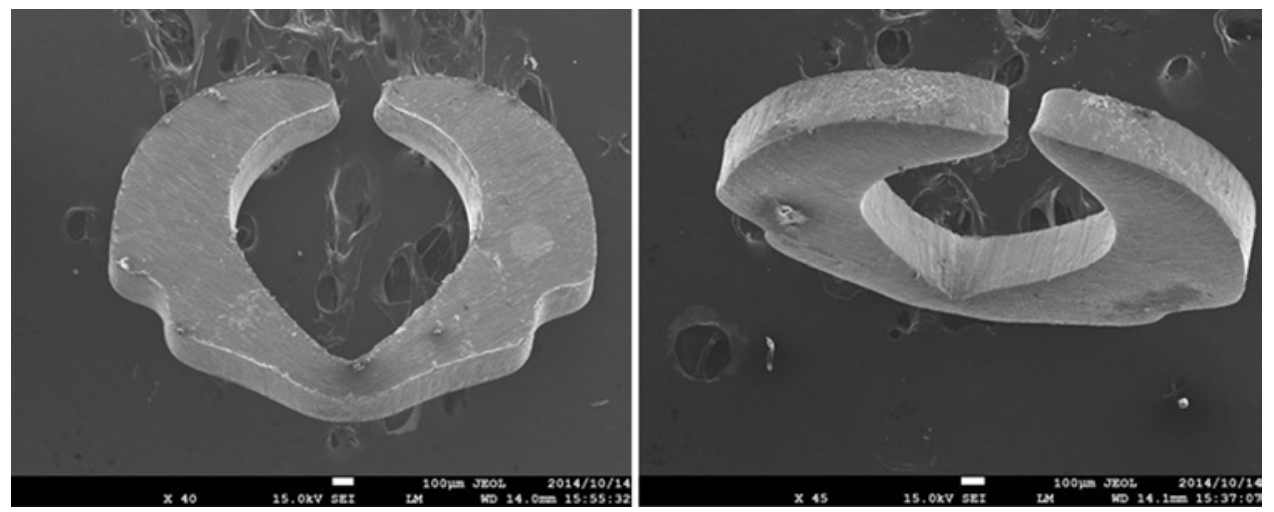

FIG. 1. Scanning electron microscopy of a nonpenetrating titanium clip showing that the tips do not completely close: lateral (left, magnification $\times 40$ ) and superior oblique (right, magnification $\times 45$ ) view of the tip. 


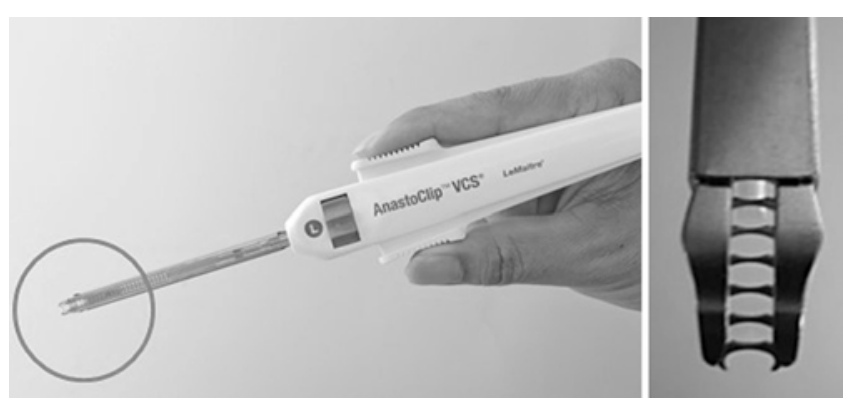

FIG. 2. Nonpenetrating titanium clip applier (left). Circle indicates the preloaded clips. Magnification of the circled image on the left (right). The clip applier allows repetitive suturing with 20 preloaded clips.

\section{Statistical Analysis}

The data were compared using the ANOVA technique, and Tukey's procedure was used for the post hoc analysis. All statistical analyses were performed using the IBM SPSS software version 20 (IBM Corp.). A p value $<0.05$ was considered statistically significant.

\section{Results}

\section{Experimental Studies}

\section{Leakage Pressure Measurements}

The mean ( \pm standard deviation) water pressures that could be sustained without leakage were $16.7 \pm 6.3 \mathrm{~mm}$ $\mathrm{Hg}$ in the ePTFE suture group and $252.0 \pm 101.2 \mathrm{~mm} \mathrm{Hg}$ in the nonpenetrating titanium clip group. A significant difference was apparent between the 2 groups, with the nonpenetrating titanium clip group showing a leakage pressure that could be sustained and was $1508 \%$ higher than that of the ePTFE suture group ( $p=0.001$; Fig. 6).

When the nonpenetrating titanium clip suture line was sealed using an absorbable PGA mesh soaked with fibrin glue, the mean leakage pressures were $297.5 \pm 26.7,279.0$ \pm 52.9 , and $449.7 \pm 65.4 \mathrm{~mm} \mathrm{Hg}$ at 5 minutes, 30 minutes, and 12 hours, respectively. No significant differences were

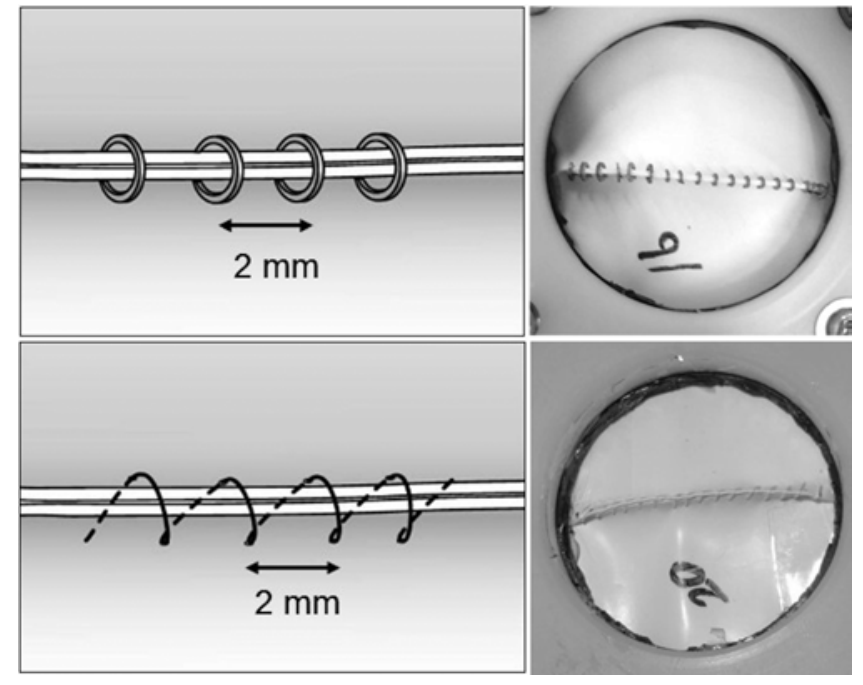

FIG. 4. Upper: Schematic and photograph of the ePTFE sheet approximated with nonpenetrating titanium clips placed in an interrupted fashion at 2-mm intervals. Lower: Schematic and photograph of the ePTFE sheet approximated with ePTFE sutures, with a needle to thread ratio of 1:1. The running sutures were at 2-mm intervals as well.

evident between the leakage pressures at 5 and 30 minutes. The differences between the leakage pressures at 5 minutes and 12 hours and at 30 minutes and 12 hours were statistically significant $(\mathrm{p}=0.001$ for both comparisons; Fig. 7). After 12 hours, the leakage pressure associated with the use of the clips combined with the mesh-and-glue technique was $180 \%$ higher than the leakage pressure of the control model that did not use the mesh-and-glue technique $(\mathrm{p}=0.001)$.

\section{Analysis of the Leakage Patterns During the Pressure Leakage Tests}

The leakage patterns were observed when the sutures in the ePTFE sheet were not covered with fibrin glue and
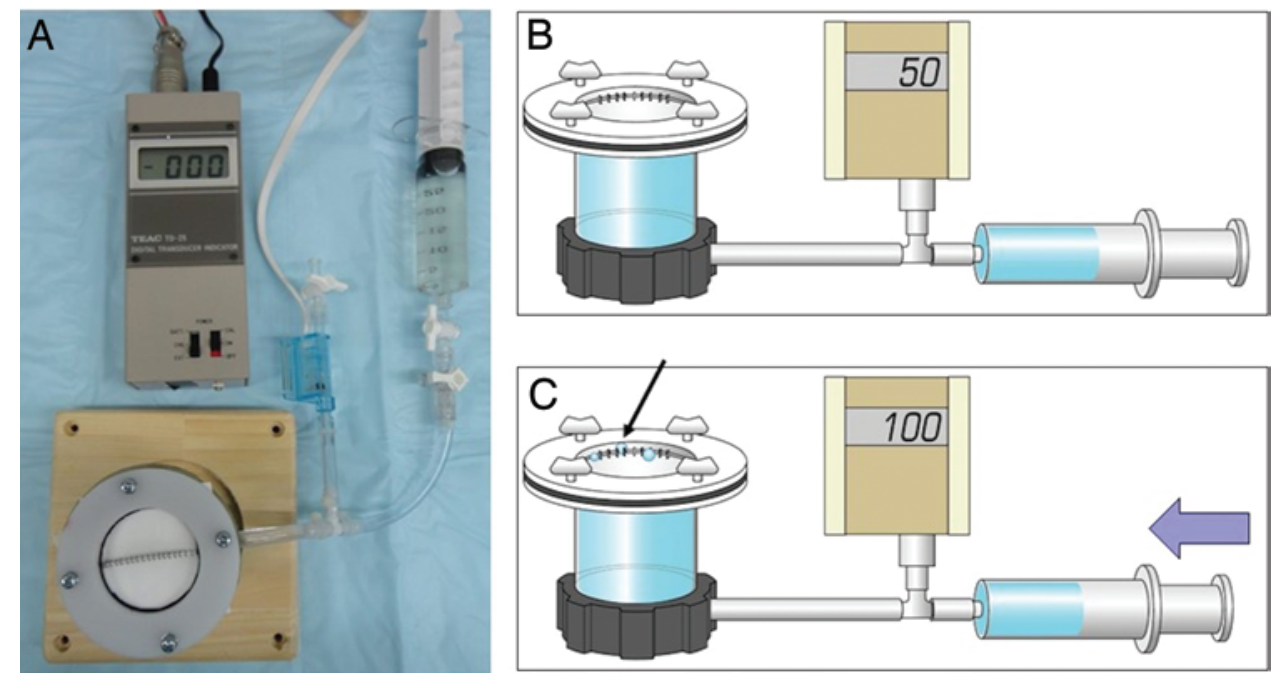

FIG. 3. A: In vitro experiment setup. The sutured ePTFE sheet covered the top of the cup. A manometer, which measured the pressure, and a syringe were attached to the cup. B: Schematic representation of the experimental setup during a pressure leakage test. C: Leakage pressure was measured based on the leakage of fluid (black arrow). 

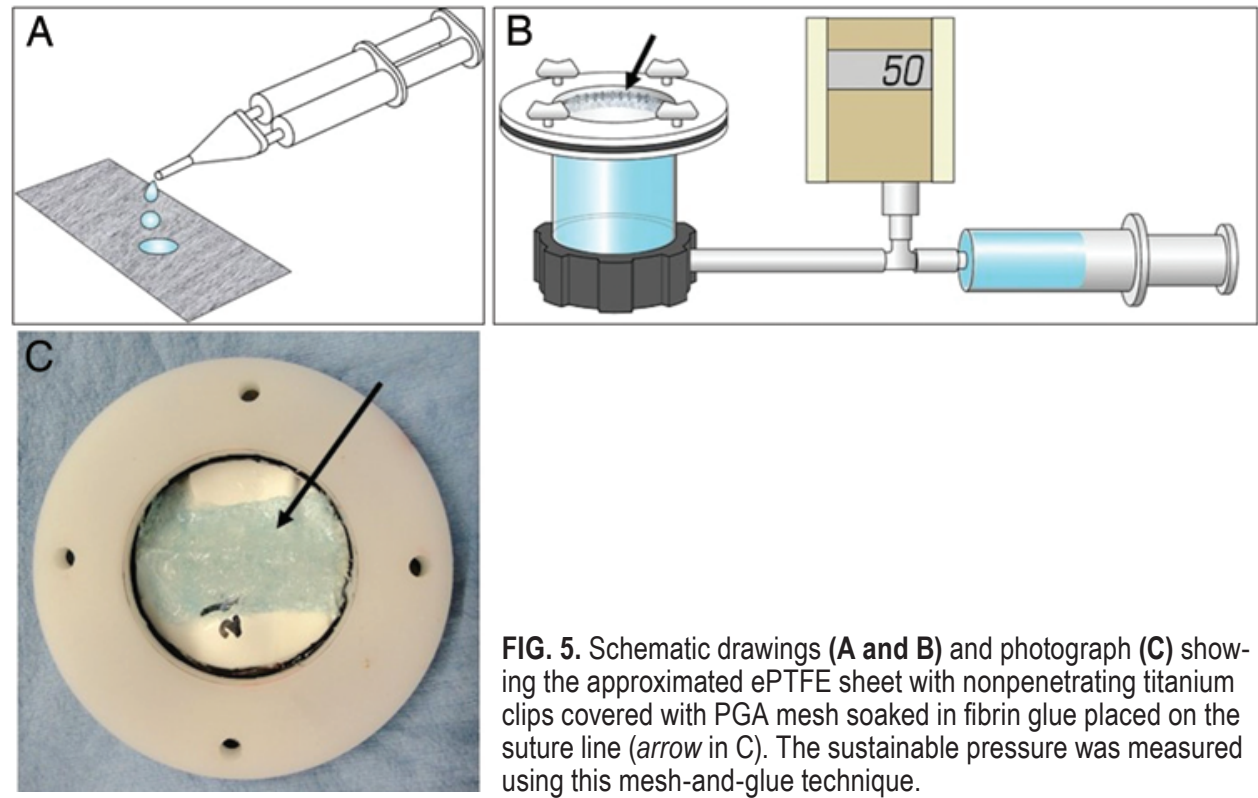

FIG. 5. Schematic drawings (A and B) and photograph (C) showing the approximated ePTFE sheet with nonpenetrating titanium clips covered with PGA mesh soaked in fibrin glue placed on the suture line (arrow in $\mathrm{C}$ ). The sustainable pressure was measured using this mesh-and-glue technique.

PGA. The nonpenetrating titanium clips did not make any suture holes in the ePTFE sheet, and fluid leakage occurred between the clips (Fig. 8 left). In the ePTFE suture group, the fluid leakage was associated with the pressure elevations and occurred at the suture holes (Fig. 8 right).

\section{Clinical Study}

Postoperative subcutaneous CSF accumulation occurred in 1 patient $(3.2 \%)$ who had a low-set conus associated with a spinal lipoma. However, conservative therapies, which included the aspiration of CSF and intravenous injections of factor XIII, improved the CSF accumulation. Intraoperatively, the spinal dura mater was very thin, and the edges of the dural incision were inadequately approxi-

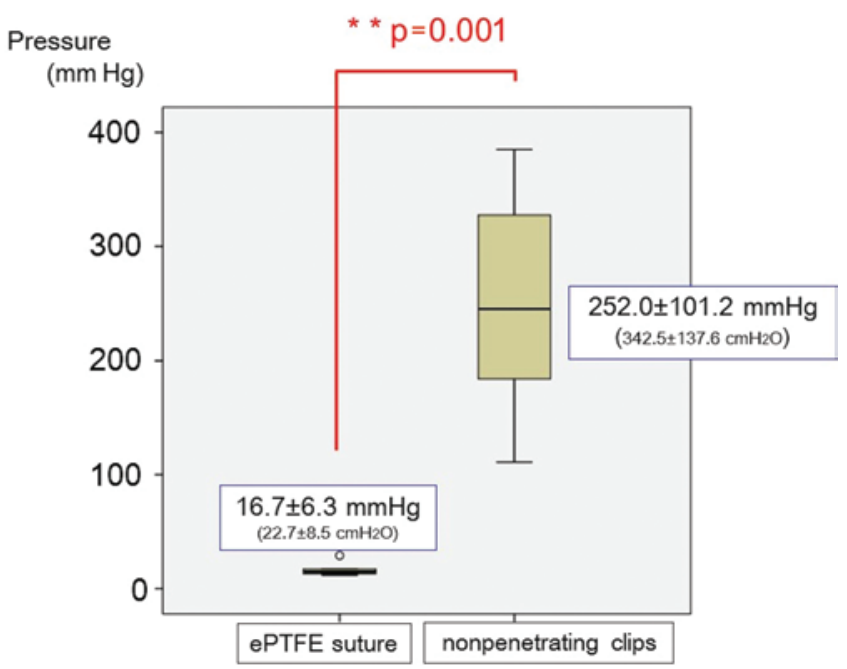

FIG. 6. The mean water pressures that could be sustained without leakage were $16.7 \pm 6.3 \mathrm{~mm} \mathrm{Hg}$ in the ePTFE suture group and 252.0 $\pm 101.2 \mathrm{~mm} \mathrm{Hg}$ in the nonpenetrating titanium clip group. A significant difference was apparent between the 2 groups, with the nonpenetrating titanium clip group showing a leakage pressure that was $1508 \%$ higher than that of the ePTFE suture group $(p=0.001)$. mated. Unlike the procedure that utilizes sutures, approximating the dura mater with the nonpenetrating titanium clips tended to insufficiently verify the suture surface. Hence, there was a fundamental need for symmetrical dural wall eversion and approximation using additional corner stitches before clip placement. We did not encounter any other complications, for example, allergic reactions, adhesions, or infections.

\section{Illustrative Case}

A 46-year-old woman was admitted to the hospital with a 4-year history of numbness and weakness in her left lower extremity. Preoperative MR images of the thoracic spine revealed a large extramedullary tumor at the T-8 to T-9 level (Fig. 9A). The tumor was located on the ventral side of the spinal cord. To obtain a workable surgical field, the left pedicle was partially removed without removing the ribs (Fig. 9B), after which the tumor was removed. The dura was sutured with nonpenetrating titanium clips, which were covered with PGA mesh and fibrin glue. Postoperative MR images of the thoracic spine showed the absence of remnant tumor and radiographic artifacts around the nonpenetrating titanium clips (Fig. 9C).

\section{Discussion}

Postoperative CSF leakage is one of the major surgical complications associated with neurosurgery. Patients with persistent CSF leaks are at risk for meningitis, arachnoiditis, and pseudomeningocele. Hence, it is critical to prevent CSF leaks, particularly after surgery for spinal intradural lesions. When the dura mater is closed during spinal surgery, it must be meticulously sutured to avoid CSF leakage that can be caused by increased hydrostatic pressure in the spine or by the patient assuming an upright position. ${ }^{4}$ However, primary dural closure is challenging when the dura mater is fragile and the operating field is too narrow and deep to allow proper stitching. 


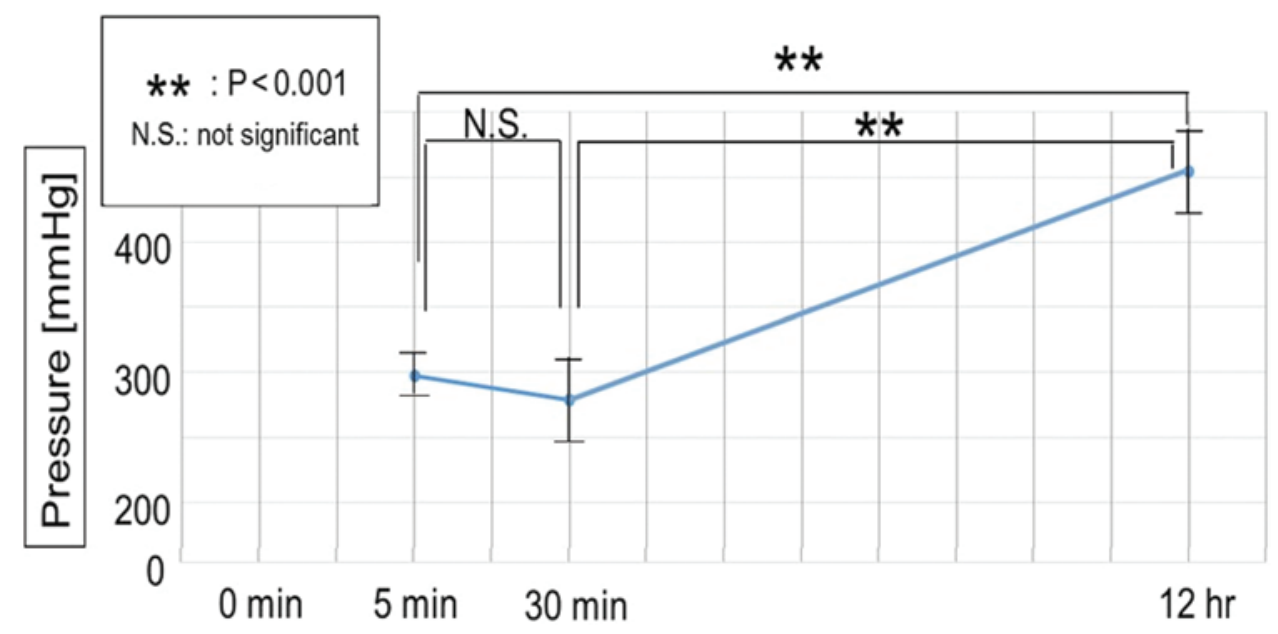

FIG. 7. The mean water pressures that could be sustained without leakage using the nonpenetrating titanium clips and the meshand-glue technique, which involves an absorbable PGA mesh soaked with fibrin glue, were $297.5 \pm 26.7,279.0 \pm 52.9$, and 449.7 $\pm 65.4 \mathrm{~mm} \mathrm{Hg}$ for 5 minutes, 30 minutes, and 12 hours, respectively. The differences between the leakage pressures at 5 minutes and 12 hours and at 30 minutes and 12 hours were statistically significant ( $p=0.001$ for both comparisons).

Several authors have described their attempts to increase the sustainable pressure after dural closure in vitro, and they have used several sealing materials and methods., ${ }^{410,14}$ The prevention of postoperative CSF leakage is very important not only in the spinal surgery field, but also following pituitary surgery. Several reports have described the prevention of CSF leakage after pituitary surgery. ${ }^{18}$ Hida et al. described a nonsuture dural repair technique, which involved the use of a dura substitute composed of PGA mesh and fibrin glue. ${ }^{4}$ An in vitro study showed that the mean threshold for water pressure without leakage was $161 \pm 42 \mathrm{~mm} \mathrm{Hg}$. Yano et al. investigated the reaction speed and the strength of the bond between PGA mesh and fibrin glue in vitro in preparation for applying this combination to pituitary surgery. ${ }^{18}$ The authors adhered PGA sheets to rabbit skin with fibrin glue within 3 minutes, and they found that this combination withstood a pressure of more than $220 \mathrm{~mm} \mathrm{Hg}$. In our study, the mean water pressure that could be retained without leakage in the presence of nonpenetrating titanium clips was $252.0 \pm$ $101.2 \mathrm{~mm} \mathrm{Hg}$, which indicated a significantly better sealing effect in the titanium clip group than in the ePTFE suture group. Indeed, the leakage pressure of the nonpenetrating titanium clip group was 15.7 times greater than that of the ePTFE suture group. In contrast to Yano et al., we used an ePTFE sheet, which is a chemically derived material, to ensure the experiment was performed under constant conditions. However, the results from our current study are not inferior to those from the previous study.

The dura mater is thinner and more fragile in the spinal region than in the intracranial region..$^{13,16,17}$ The cranial dura mater is composed of 2 layers, namely, the periosteal and the meningeal layer. The orientation of the collagen fibers differs within each layer (Fig. 10). Within the spinal column, the outer layer becomes the periosteum of the vertebral canal and the anterior and posterior atlantooccipital membranes, which are separated from the spinal dura mater. ${ }^{17}$ Hence, the spinal dura mater is a single meningeal layer that encloses collagen fibers that are in the same orientation, and as a consequence of the longitudinal direction of the collagen fibers within the spinal dura mater, it tends to tear easily. It is well established that the physical characteristics of the human dura mater, for example, its tensile strength and resistance to tearing forces, depend on the orientation of the collagen fibers. ${ }^{13}$ The spinal dura mater tears easily during suturing with surgical needles and threads. Moreover, the fluid leaked from the suture holes in our experiment. The ePTFE suture CV-5 was prepared with a needle/thread ratio of 1:1 to minimize needle-hole leakage during dural suturing. However, fluid leaks were observed in the suture holes (Fig. 11). In contrast, the nonpenetrating titanium clips did not create needle holes during dural closure, and fluid leakage occurred along the suture line between the clips. Given the structure of the spinal dura mater, it is possible that dural lacerations are created next to the needle holes and that spinal fluid could leak from the needle holes and dural lacerations. Therefore, nonpenetrating titanium clips, which do not create any suture holes, are considered ideal for approximating the spinal dura mater while it is being sutured. Indeed, only 1 patient (3.2\%) in our clinical series
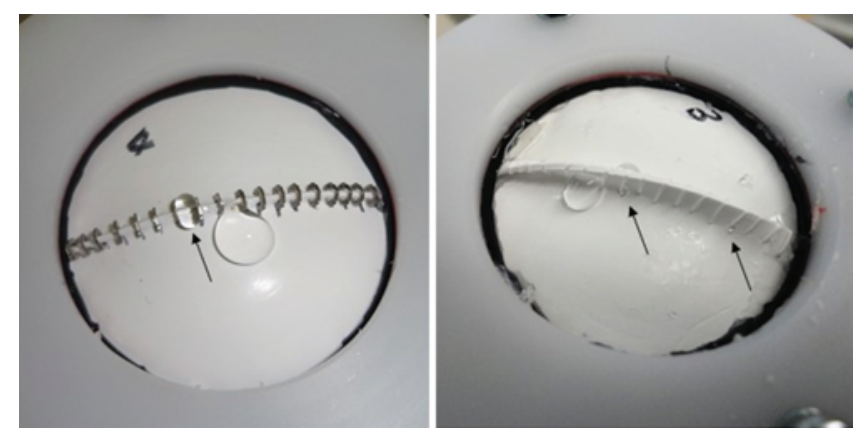

FIG. 8. Leakage patterns without coverings of fibrin glue and PGA. Left: The nonpenetrating titanium clips did not create suture holes, and fluid leakage was confirmed between the clips (arrow). Right: In the ePTFE suture group, fluid leakage was associated with pressure elevations and occurred at the suture holes (arrows). 


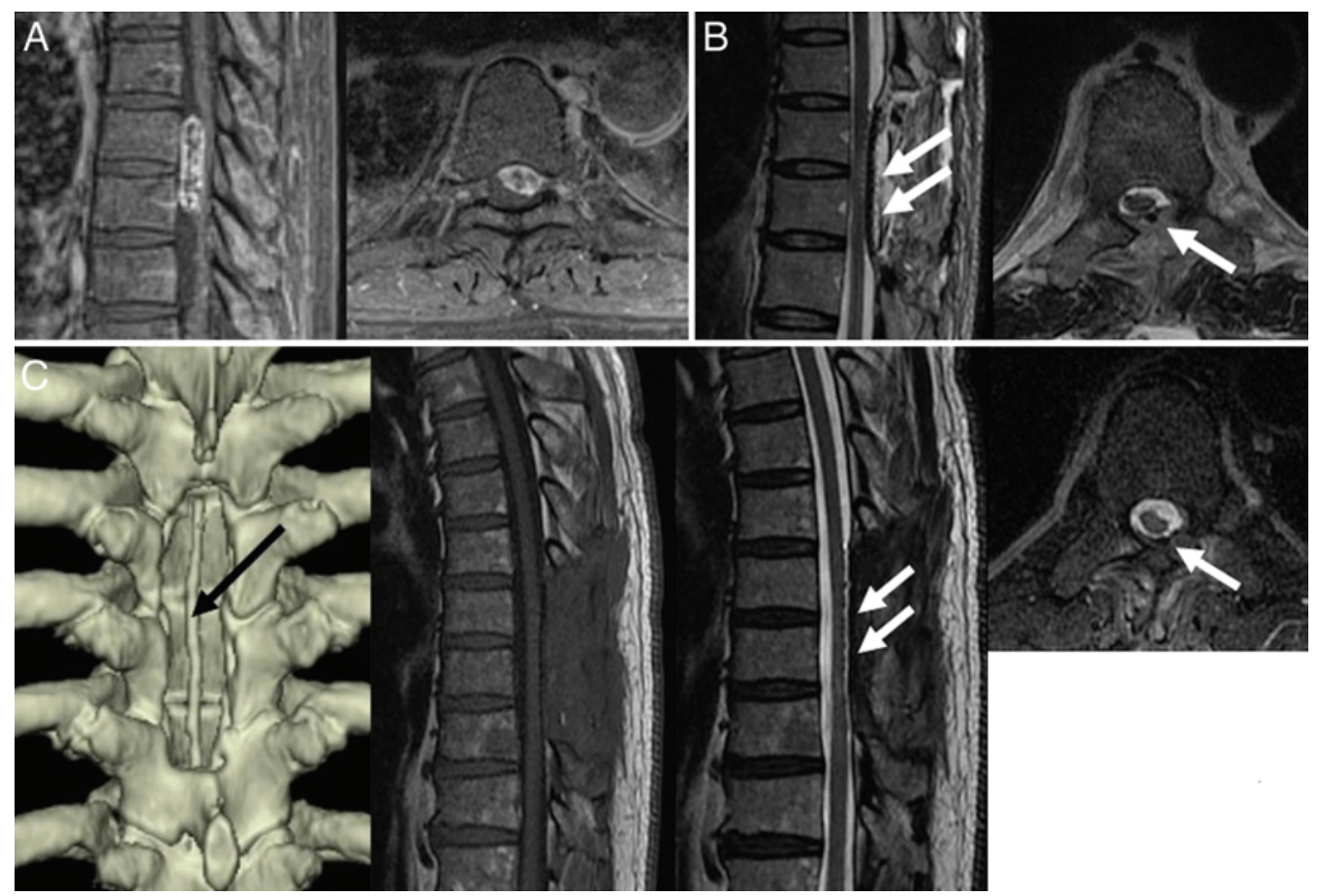

FIG. 9. A: Preoperative T1-weighted MR images with gadolinium enhancement showed a ventrally located lesion at the level of T-8 and T-9. B: Postoperative MR images taken 2 weeks after surgery in which there is no leakage of the CSF, evidence of remnant tumor, or mechanical artifacts caused by the nonpenetrating titanium clips (white arrows) used for dural closure. C: A postoperative CT scan (left) taken 4 months after surgery, showing the clips (black arrow). Postoperative MR images (right) of the thoracic spine taken 4 months after surgery, demonstrating the absence of remnant tumor and no mechanical artifacts (white arrows).

experienced a postoperative subcutaneous accumulation of CSF. Furthermore, when the nonpenetrating titanium clip suture line was sealed using the mesh-and-glue technique, the mean leakage pressure was $449.7 \pm 65.4 \mathrm{~mm} \mathrm{Hg}$ at 12 hours. The leakage pressure of the nonpenetrating titanium clip group was 1.8 times higher than that of the ePTFE suture group.

When we compared results from experiments that used the nonpenetrating titanium clips alone with those from experiments that used the nonpenetrating titanium clips in

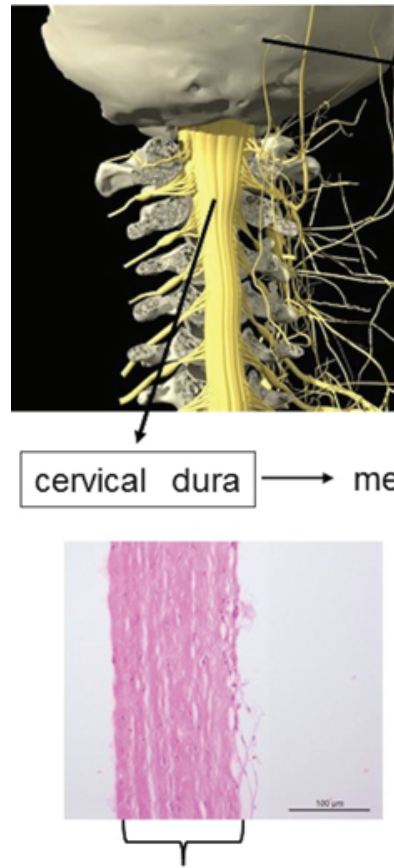

inner layer
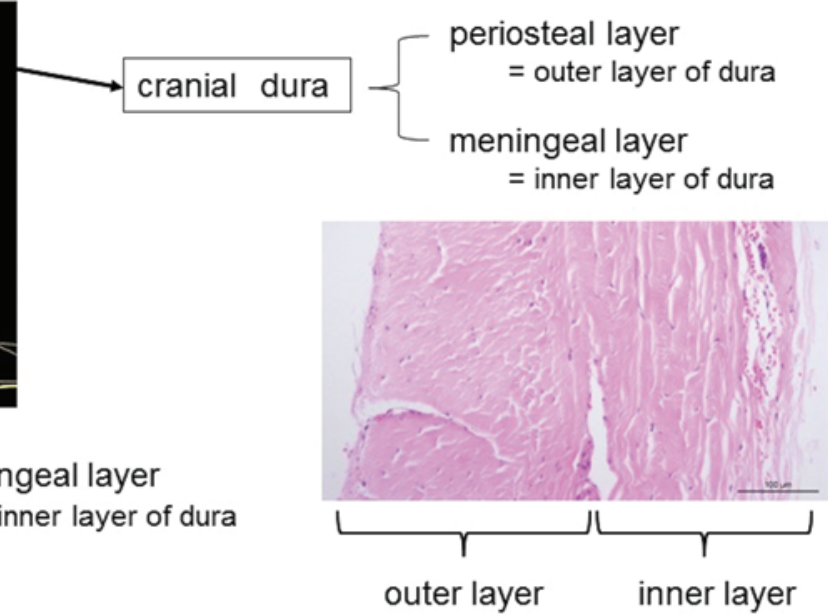

FIG. 10. An H \& E-stained histological specimen of the cranial dura mater (right), which is composed of 2 layers histologically, namely, the periosteal and meningeal layers. Each layer contains differently oriented collagen fibers. The outer layer becomes the periosteum at the craniovertebral junction. The spinal dura mater is a single meningeal layer, which encloses collagen fibers with the same orientation as shown in the $\mathrm{H}$ \& E-stained specimen (left). Bar in photomicrographs $=100 \mu \mathrm{m}$. 


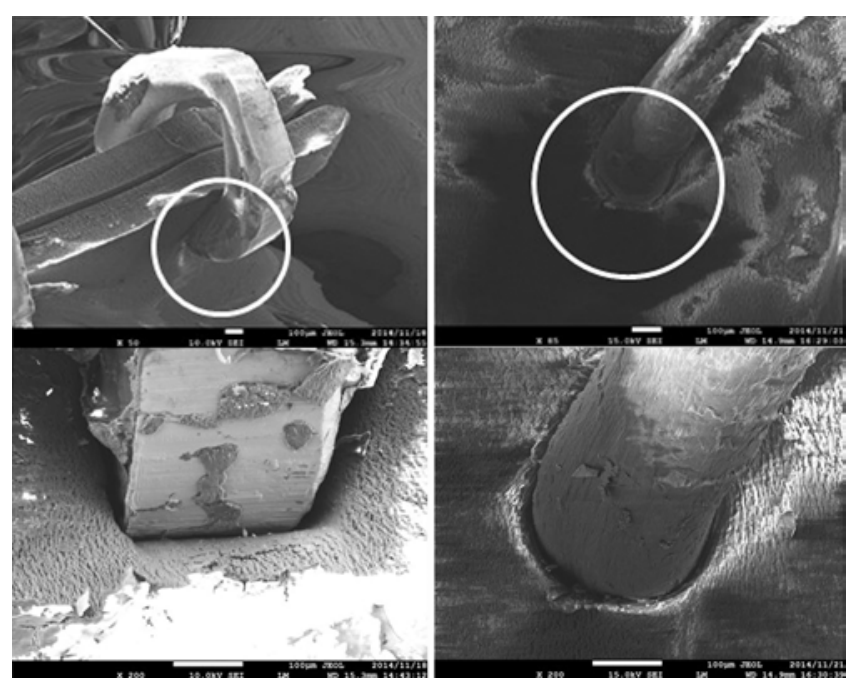

FIG. 11. Scanning electron micrograph of nonpenetrating titanium clips applied to the ePTFE sheet at low magnification (upper left) and high magnification (lower left). The tip of the clip was fixed to the ePTFE sheet but had not penetrated the sheet, and no suture holes were apparent. The ePTFE suture (CV-5, needle/thread ratio 1.0) was used for dural closure, and scanning electron micrographs showed that the suture hole was almost filled with thread and that the gap between the thread and sheet was very small (upper and lower right). Original magnification $\times 50$ (upper) and $\times 200$ (lower).

combination with the PGA mesh and fibrin glue, we found that the mean leakage pressures were $252.0 \pm 101.2$ and $449.7 \pm 65.4 \mathrm{~mm} \mathrm{Hg}$, respectively. These findings indicate that using the nonpenetrating titanium clips with the fibrin glue and the absorbable PGA mesh could create a strong membrane, which would tolerate high pressures if it could be tightly attached to the surrounding structures. As in a previous study, ${ }^{9}$ the mesh-and-glue technique was also useful for approximating the ePTFE sheet with the nonpenetrating titanium clips. Moreover, contrary to expectations, the MR images taken after surgery showed no mechanical artifacts caused by the nonpenetrating titanium clips used for dural closure. Hence, this clip is suitable for surgery involving the removal of intradural tumors.

Cost is one concern associated with the use of nonpenetrating titanium clips. However, reported benefits may offset the greater cost of these clips. In experimental studies, some authors have noted differences between nonpenetrating titanium clips and conventional sutures as regards the time taken to suture vascular walls, $, 1,2,7,12,19$ and the mean vascular suturing time was significantly shorter when nonpenetrating titanium clips were applied, as compared with the time required to apply sutures. One study reported that nonpenetrating titanium clips saved up to $88 \%$ of the vascular suturing time compared with conventional sutures.? Hence, using nonpenetrating titanium clips may shorten operative times.

Recently, minimally invasive procedures have been introduced to spinal surgery. We also perform lumbar discectomies using percutaneous endoscopic procedures in the narrow deep-seated surgical field. While we have experienced dural tears during these surgical procedures, we have not yet used nonpenetrating titanium clips. In the scope of our future research, nonpenetrating titanium clips have potential for use in narrow surgical fields, including in the anterior cervical approach, skull base surgery, and pituitary surgery. We consider the use of nonpenetrating titanium clips in these procedures as our next challenge.

There are limitations to this study. In the clinical context, additional factors, including the impact of the CSF pulse, continuous CSF flow during fibrin glue application, surrounding adipose tissue, collagen fibers of the spinal dura mater, and sudden variations in the intracranial pressure, must be considered. In the present study, however, we aimed to obtain fundamental information about the strength of nonpenetrating titanium clips before we accounted for these variations.

\section{Conclusions}

The interrupted placement of nonpenetrating titanium clips enables dural closure without creating any holes. These clips facilitate improvements in the initial leakage pressure and reduce postoperative CSF leakage following spinal surgery. We conclude that it is very beneficial to suture the spinal dura mater with nonpenetrating titanium clips given the anatomical characteristics of the spinal dura mater and the fact that the clips do not create suture holes.

\section{Acknowledgments}

We thank Professor K. Oyanagi, Ms. M. Asakawa, and Ms. A. Nakahara at the Division of Neuropathology, Department of Brain Disease Research; Dr. K. Kametani at the Department of Instrumental Analysis, Research Center for Human and Environmental Sciences, Shinshu University School of Medicine; and Drs. T. Hashimoto and K. Higuchi at the Department of Neurology, Aizawa Hospital, for their technical support.

\section{References}

1. Dimakakos PB, Pafiti-Kondi A, Doufas A, Kotsis T, Mourikis D, Rizos D: Venous repair with vascular clips and conventional suture: a comparative experimental study. Phlebology 14:65-70, 1999

2. Ducasse E, Basseau F, Puppinck P, Baquey C: [Can minimal arterial aggressions using non-penetrating mechanical clip suture prevent myo-intimal hyperplasia? Preliminary results.] J Mal Vasc 26:50-54, 2001 (Fr)

3. Gaskill DM, Stewart S, Kirsch WM, Zhu YH, Thompson K: Sutureless vasovasostomy: new technique using experimental microclip in rat model. Urology 40:191-194, 1992

4. Hida K, Yamaguchi S, Seki T, Yano S, Akino M, Terasaka $S$, et al: Nonsuture dural repair using polyglycolic acid mesh and fibrin glue: clinical application to spinal surgery. Surg Neurol 65:136-143, 2006

5. Kirsch WM, Zhu YH, Hardesty RA, Chapolini R: A new method for microvascular anastomosis: report of experimental and clinical research. Am Surg 58:722-727, 1992

6. Kirsch WM, Zhu YH, Hardesty RA, Petti G, Furnas D: Nonpenetrating clips successfully replacing sutures in base of skull surgery. Skull Base Surg 3:171-181, 1993

7. Leppäniemi A, Wherry D, Pikoulis E, Hufnagel H, Waasdorp C, Fishback N, et al: Arterial and venous repair with vascular clips: comparison with suture closure. J Vasc Surg 26:2428, 1997

8. Levy DI, Sonntag VK: Titanium dural clip testing. Technical note. J Neurosurg 81:947-949, 1994 
9. Nagata K, Kawamoto S, Sashida J, Abe T, Mukasa A, Imaizumi Y: Mesh-and-glue technique to prevent leakage of cerebrospinal fluid after implantation of expanded polytetrafluoroethylene dura substitute-technical note. Neurol Med Chir (Tokyo) 39:316-319, 1999

10. Nakajima S, Fukuda T, Hasue M, Sengoku Y, Haraoka J, Uchida T: New technique for application of fibrin sealant: rubbing method devised to prevent cerebrospinal fluid leakage from dura mater sites repaired with expanded polytetrafluoroethylene surgical membranes. Neurosurgery 49:117123, 2001

11. Palm SJ, Kirsch WM, Zhu YH, Peckham N, Kihara S, Anton $\mathrm{R}$, et al: Dural closure with nonpenetrating clips prevents meningoneural adhesions: an experimental study in dogs. Neurosurgery 45:875-882, 1999

12. Pikoulis E, Rhee P, Nishibe T, Burris D, Leppäniemi AK, Fishback N, et al: Arterial repair with synthetic patch by using titanium clips. J Trauma 48:292-295, 2000

13. Protasoni M, Sangiorgi S, Cividini A, Culuvaris GT, Tomei G, Dell'Orbo C, et al: The collagenic architecture of human dura mater. J Neurosurg 114:1723-1730, 2011

14. Shaffrey CI, Spotnitz WD, Shaffrey ME, Jane JA: Neurosurgical applications of fibrin glue: augmentation of dural closure in 134 patients. Neurosurgery 26:207-210, 1990

15. Shibata T, Suehiro S, Sasaki Y, Hattori K, Fujioka M, Kumano H, et al: Use of a vascular closure system for bypass grafting of peripheral arteries. Vasc Endovascular Surg 32:315-320, 1998

16. Shimizu T: [Surgical approach and related anatomy of posterior craniocervical region.] Spine and Spinal Cord 17:411413, 2004 (Jpn)

17. Standring S: Gray's Anatomy: The Anatomical Basis of Clinical Practice, ed 40. Edinburgh: Churchill Livingstone/ Elsevier, 2008
18. Yano S, Tsuiki H, Kudo M, Kai Y, Morioka M, Takeshima H, et al: Sellar repair with resorbable polyglactin acid sheet and fibrin glue in endoscopic endonasal transsphenoidal surgery. Surg Neurol 67:59-64, 2007

19. Zeebregts C, van den Dungen J, Buikema H, van der Want J, van Schilfgaarde R: Preservation of endothelial integrity and function in experimental vascular anastomosis with nonpenetrating clips. Br J Surg 88:1201-1208, 2001

20. Zhu YH, Kirsch WM, Cushman R, Becker K, McCabe W, Kornfeld M, et al: Comparison of suture and clip for microvascular anastomoses. Surg Forum 36:492-495, 1985

\section{Disclosure}

The authors report no conflict of interest concerning the materials or methods used in this study or the findings specified in this paper.

\section{Author Contributions}

Conception and design: Ito. Acquisition of data: Ito. Analysis and interpretation of data: Ito. Drafting the article: Ito. Reviewed submitted version of manuscript: Horiuchi. Approved the final version of the manuscript on behalf of all authors: Ito. Statistical analysis: Ito, Aoyama, Horiuchi. Administrative/technical/material support: Aoyama, Horiuchi, Hongo. Study supervision: Hongo.

\section{Correspondence}

Kiyoshi Ito, Department of Neurosurgery, Shinshu University School of Medicine, 3-1-1 Asahi, Matsumoto 390-8621, Japan. email: kitoh@shinshu-u.ac.jp. 